\title{
Situación actual de las Vacunas e Inmunización en Haití
}

\author{
Marc-Henry Syldora
}

\begin{abstract}
a Residente de Medicina Familiar y Comunitaria. Centro de Salud Cuenca I, Cuenca.

\section{Correspondencia:}

Marc-Henry Syldor,

c/ Teniente Benítez n $7,3^{\circ} \mathrm{B}$, 16003 - Cuenca (España). Telf.: 697462146,

e-mail: msyldor@yahoo.fr

Recibido el 10 de enero de 2009.
\end{abstract}

Aceptado para su publicación el 17 de febrero de 2009.

\section{RESUMEN}

La inmunización ha sido uno de los grandes éxitos de la salud pública. Ha permitido salvar la vida de millones de niños en Haití y le ha dado a miles más la oportunidad de vivir una vida más larga y saludable, al igual que mayores oportunidades de instruirse, de leer y escribir, de jugar y de moverse libremente sin sufrimiento. No obstante, existen deficiencias en la cobertura, en la prestación de servicios de salud, en la seguridad de las vacunas y en el acceso a nuevas vacunas del Programa Ampliado de Inmunización (PAI), entre otras. La cobertura vacunal no supera el $40 \%$ como promedio nacional, alcanzando sólo al $18 \%$ en algunos departamentos.

Palabras clave: Vacunas, Cobertura vacunal.

\section{ABSTRACT}

Vaccines and Immunisation - current situation in Haiti

Immunisation is one of the great public health successes. It has saved millions of children's lives in Haiti and has given thousands more the opportunity to live a long and healthy life, to become educated, to learn to read and write, to play and move about freely without suffering. However, there are deficiencies in coverage levels, in healthcare provision, in vaccine safety and in access to the new vaccines of the Expanded Programme on Immunisation (EPI), amongst others. Vaccine coverage did not exceed $40 \%$ as a national average, reaching only $18 \%$ in some areas.

Key words. Vaccines. Immunization Coverage.

\section{INTRODUCCIÓN}

Las vacunas han cambiado la historia y el desarrollo humano, constituyendo una verdadera revolución de salud pública. Los últimos descubrimientos permiten mantener grandes esperanzas. En Haití se practica hoy en día la vacunación sistemática contra el sarampión, la polio, la difteria, el tétanos, la tos ferina y la tuberculosis, adoptando el esquema recomendado por la Organización Mundial de la Salud (OMS). Por lo general, los niños más pobres tienen acceso a una gama más reducida de vacunas y están más expuestos a los peligros de las prácticas de vacunación poco seguras. Los servicios de inmunización del país adolecen de insuficiente compromiso político y de escasas inversiones, al tiempo que los programas de inmunización padecen las deficiencias de los sistemas de prestación de servicios de salud.

Haití es la nación más pobre de América Latina con 480 \$US de renta, con una población estimada de 8,6 millones de habitantes ${ }^{1}$. La mayor parte de la población depende del Programa Ampliado de Inmunización (PAI), aunque existe una minoría en la capital que tiene acceso a una gama más amplia de vacunas para sus niños. Los beneficios de la inmunización no han llegado por igual a todos los niños del país. Cada vez son más los que, por el solo hecho de haber nacido en determinado lugar, quedar sistemáticamente excluidos de esos beneficios.

El objetivo de este trabajo es presentar la situación actual de las vacunas e inmunización en Haití, sus logros, así como los desafíos y su impacto en el estado de salud de la población. 


\section{HISTORIA DEL PAI EN HAITÍ}

La inmunización representa uno de los grandes logros de la salud pública del siglo XX. Desde el lanzamiento del PAl, en 1974, los programas nacionales de vacunación de todo el mundo han conseguido evitar millones de muertes. Fue implantado en Haití en los años ochenta. El PAI tiene dos grandes grupos principales: 1. Los niños de menos de un año, que constituyen alrededor del 3,5\% de la población total.

2. Las mujeres sexualmente activas (15-49 años). Este grupo representa alrededor del $25 \%$ de la población general, incluyendo también a las mujeres embarazadas ( $4 \%$ de la población total).

Los niños de menos de un año deben ser completamente vacunados contra 6 enfermedades inmunoprevenibles antes de cumplir su primer año de nacimiento. Las mujeres sexualmente activas, incluyendo a las gestantes, deben recibir tres dosis contra el tétanos para ser inmunizadas de por vida. Hay una actividad destinada a los niños de 1 y 5 años cuyo estatus vacunal no ha sido completado durante su primer año de vida². Además, se aprovecha la administración sistemática de vacunas para la prestación de otras intervenciones sanitarias, tales como la administración de suplementos de vitamina A, destinada a prevenir trastornos nutricionales.

La inmunización se practica en la mayoría de las instituciones sanitarias del país (80\%), pero no de forma regular. Las instituciones que ofrecen el mayor número de servicios de vacunación son los dispensarios públicos (24\%). La debilidad y la insuficiencia de los equipos para almacenamiento es un reto para la regularidad de los servicios de vacunación. La ruptura de la llegada del gas propano, jeringuillas y otros accesorios esenciales es recurrente. A pesar de esta situación, importantes logros han sido obtenidos ${ }^{3}$.

\section{SITUACIÓN DE LAS ENFERMEDADES PREVENIBLES POR VACUNAS (EPV)}

Hay una reducción evidente de las enfermedades del PAI, pero la cobertura es aún insuficiente en Haití. La

\begin{tabular}{llllll}
\hline \multicolumn{4}{l}{ Esquema de Vacunación, 2006 (HAITI) } & & \\
\hline BCG & RN & & & & \\
VOP & RN & $6 s$ & $10 s$ & $14 s$ & $+1^{a}$ \\
DTP & RN & $6 s$ & $10 s$ & $14 s$ & $+1^{a}$ \\
Sarampión & $9 \mathrm{~m}$ & & & & \\
TT/Td & MEF & MEF & MEF & MEF & MEF \\
\hline
\end{tabular}

Tabla 1. Esquema de vacunación 2006 (Haitî). RN: recién nacido; MEF: mujeres en edad fértil. cobertura vacunal de 12 a 23 meses es del $41 \%$ y el $11 \%$ los niños no han recibido ninguno de las vacunas del PAI. Hay un $48 \%$ de niños que están parcialmente vacunados (EMMUS IV) 4 . El $11 \%$ de niños que no tienen acceso a la vacunación son susceptibles de contraer una enfermedad evitable. Las carreteras deficientes constituyen un enorme obstáculo logístico a la hora de las distribuciones de vacunas. Muchas de las localidades sufren de una baja cobertura vacunal. El alejamiento de las instituciones sanitarias, la irregularidad de los servicios de vacunación y la insuficiencia de las estrategias de extensión de cobertura destinadas a las poblaciones lejanas o enclavadas representan los principales problemas del PAl, que comenzó al principio del mes de noviembre de este año la más extensa campaña de su historia. Esta campaña de vacunación en masa permitirá erradicar la rubéola y el sarampión, y prevenir toda reaparición y mutación del virus de la poliomielitis. Se movilizó aproximadamente a 8.328 agentes de salud y se incorporó a unos 3.545 cooperantes, con el fin de permitir que todos los niños y adultos estimados por la campaña estén vacunados. Esta campaña se organizó con el fin de asegurar la efectividad de la estrategia y las herramientas necesarias para una campaña nacional que afectará todo el territorio durante 3 meses. En esta campaña Haití va a introducir la vacuna bivalente sarampión/ rubéola (RR) en su programa ${ }^{5}$. Se constata que hay una importante pérdida de la cobertura vacunal del DPT, pues, aunque hay un $83 \%$ que recibe la primera dosis, sólo un $53 \%$ son completamente vacunados con las tres dosis. El sarampión esta a punto de erradicarse. Un total de 990 casos de sarampión fueron confirmados en el año 2000. La ultima epidemia se remonta al año 2001, con 158 casos, no habiéndose confirmado ningún otro caso desde ese momento.

En Haití un solo se ha confirmado un caso de poliomielitis tipo I en un niño de 2 años que fue vacunado de manera inadecuada. La parálisis fue declarada el 30 de agosto de 2000. A pesar de un trabajo intensivo de tamizaje, ningún otro caso ha sido reportado.

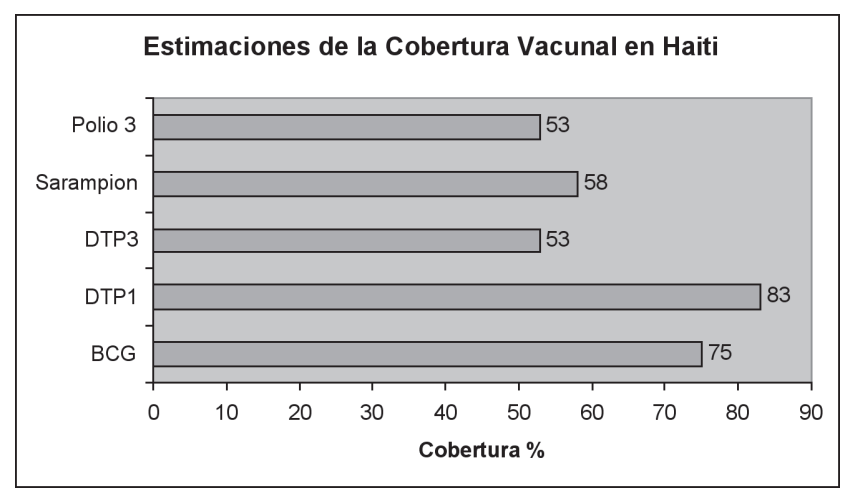

Figura 1. Eestimaciones de la cobertura vacunal en Haití. Fuente: WHO/UNICEF, 2006. 
En 2004, Haití conoció igualmente una epidemia de difteria, con 801 casos sospechosos. En 2005, los datos disponibles del Ministerio de Salud Publica y de la Población haitiana (MSPP) indican que 495 casos fueron señalados para ese mismo año (9 fueron confirmados y 8 fallecieron).

El tétanos neonatal sigue siendo un problema muy grave de salud pública en Haití. A pesar de los esfuerzos concertados para controlar esta enfermedad, 119 casos fueron señalados en 2005. Hoy en día, en algunas comunas se está registrando más de 1 caso por 1000 nacidos vivos. Según la encuesta de morbi-mortalidad y utilización de servicios de 2005-2006 (EMMUS IV), en casi tres cuartas partes de los nacidos en los últimos 5 años la madre había recibido una dosis contra el tétanos durante su embarazo.

En el caso de la tos ferina todos los casos no están señalados. Los datos disponibles fueron 4 casos en 2004 y, entre los 697 señalados por el MSPP en 2005, 21 fueron objeto de estudio.

\section{COBERTURAS DE VACUNACIÓN}

La cobertura vacunal representa la proporción de niños menores de 1 año que han recibido una dosis de BCG y están vacunados contra difteria, tétanos, tos ferina (DPT), poliomielitis (VOP) y sarampión. El porcentaje de mujeres embarazadas que han recibido dos dosis de vacuna antitetánica (TT/Td) es declarado en relación al control del tétanos neonatal (tabla 1).

Tuberculosis (BCG). El rol de la vacuna BCG en la disminución de las formas diseminadas de la tuberculosis en el niño menor es indiscutible, aunque la tuberculosis continúa afectando en forma importante a la población. La BCG es habitualmente considerada como un indicador de accesibilidad a la vacunación, ya que se administra lo mas cercano posible del nacimiento. Su cobertura ha sido siempre la más elevada de todas las vacunas del país. Hoy en día tiene una cobertura de $75 \%{ }^{6}$.

Poliomielitis. Solamente se incluyen en la evaluación de la cobertura vacunal las cifras de vacunaciones administradas en los programas de rutina, reflejando que la tasa de vacunación contra la poliomielitis sea casi idéntica a la de tres dosis de DTP. Haití tiene una cobertura vacunal para tres dosis de vacuna frente a poliomielitis del $53 \%$. De hecho, muchos niños han recibido dosis suplementarias por las campañas nacionales de inmunización, pero no se tienen en cuenta en las estadísticas.

Tos-Ferina. Entre las 6 enfermedades objeto del PAI, la tos ferina es una de las más difíciles de abordar. Existen varias razones para ello, incluyendo: dificultad en el diagnóstico, lo cual entorpece la confirmación de casos, falta de uniformidad en la definición de caso, dada la ocurrencia de formas clínicas atípicas de difícil reconocimiento y confirmación, la existencia de otras infecciones que tienen gran semejanza en su presentación clínica y subregistro de casos por las razones antes señaladas.

Sarampión. Es la enfermedad prevenible que ha matado a más niños en Haití, ya que es muy contagiosa y da lugar a epidemias explosivas. Actualmente la cobertura de vacunación sistemática contra el sarampión es del $58 \%$ La vacuna antisarampionosa se administra a los 9 meses, después de la serie inicial de inmunizaciones, y las tasas de abandono son muy elevadas.

Tétanos materno y neonatal. El tétanos es una enfermedad vinculada a la pobreza que existe en el país. Aparece durante las primeras semanas de vida, cuando el niño es particularmente vulnerable. La infección suele comenzar cuando el muñón umbilical se contamina con esporas del tétanos, normalmente por el contacto con manos sucias, la utilización de un instrumento sucio para cortar el cordón o la aplicación de material contaminado al muñón. La administración a la mujeres embrazadas de tres dosis de toxoide tetanico ofrece una protección total contra el tétanos maternal y neonatal. Una madre protegida contra el tétanos comunicara su inmunidad a su recién nacido, el cual la conservará durante dos o tres meses. A continuación, se deben administrar al niño tres dosis de DTP, dirigiéndose a la vacunación sistemática. Hoy en día, menos de una de cada tres mujeres en edad de procrear está inmunizada en Haití. La mayoría de los partos se hacen en casa con falta de condiciones higiénicas, y la cobertura global de inmunización es alrededor del $13 \%$ en la mujeres y del $79 \%$ en los niños ${ }^{7}$. El tétanos nunca podrá ser erradicado, pues las esporas de la bacteria que provoca la enfermedad (Clostridium tetani) sobreviven en el entorno aun sin contacto humano. La inmunización sistemática y la vigilancia de la enfermedad habrán de continuar, sumadas a los esfuerzos por promover partos en condiciones higiénicas (figura 1).

\section{DEFICIENCIAS DE COBERTURA}

Las diferencias de acceso a las vacunas y a la inmunización siguen menoscabando el principio de equidad en el que debería basarse el programa nacional de inmunización. En general, las personas que quedan al margen del programa de vacunación sistemática son las que viven en lugares aislados, barriadas pobres en las ciudades y zonas fronterizas. También se incluye a poblaciones desplazadas, personas sin acceso a la vacunación a causa de distintas barreras sociales, personas que carecen de información o de motivación y personas que se niegan a vacunarse.

La cobertura vacunal varía fuertemente según el lugar de residencia: son los niños de ciertas ciudades los que han recibido más frecuentemente todos las va- 
cunas (48\%), seguidos de los del Área metropolitana $(41 \%)$ y de los de la zona rural (40\%) (EMMUS IV). A pesar del éxito general de los programas de inmunización, cada año mueren alrededor de 31.000 niños menores de cinco años ${ }^{8}$. La inmunización con las vacunas actualmente disponibles podría no sólo evitar muchas de esas defunciones infantiles, sino también reducir la carga de discapacidad, enfermedad y absentismo escolar entre los niños que sobreviven.

En Haití, la mayor parte de los niños que no son vacunados quedan atrapados en lugares remotos a los que no llega ningún servicio de salud.

Es cierto que la ultima epidemia de sarampión en Haití se remonta a 6 años, lo que significa que hay una interrupción de la transmisión autóctona del sarampión en el país, pero la cobertura de inmunización contra esta enfermedad es peligrosamente baja y sabemos que el sarampión es sumamente contagioso, pudiendo propagarse rápidamente entre los niños que no han sido vacunados.

En el mismo país existen desigualdades enormes entre las poblaciones más pobres y las más ricas. Las tasas de abandono de la vacunación son particularmente altas entre las poblaciones más pobres, que no terminan de recibir todas las dosis de vacunas por dificultades de acceso a los servicios de salud o por la prestación irregular de esos servicios. Haití es un país pobre que presenta unas bajas tasas globales de cobertura vacunal.

\section{DEFICIENCIAS EN LA PRESTACIÓN DE SERVICIOS DE SALUD}

En Haití, los sistemas de prestación de servicios de salud no funcionan debidamente y el gobierno no puede atender las necesidades sanitarias básicas de la población.

La falta de inmunización sistemática de los niños es debida a que los sistemas de prestación de servicios de salud son indebidamente gestionados y equipados. Además, los servicios de salud son muy limitados, apenas existen fuera de las zonas urbanas, y las instalaciones, los vehículos y el equipo básico de la cadena de frío están mal conservados o averiados.

La capacidad para prestar servicios de salud, incluida la inmunización, también puede verse comprometida por deficiencias de gestión, escasa motivación del personal y falta de planificación y elaboración de presupuestos. Además, la ausencia de sistemas efectivos de vigilancia y notificación de enfermedades menoscaba la eficacia de los programas de inmunización y el control de las enfermedades, e impide orientar los servicios de salud hacia quienes más los necesitan.

En Haití, las comunicaciones y las infraestructuras están tan poco desarrolladas que la mayor parte de la población no tiene acceso a servicios de salud, y mucho menos a la inmunización. Al mismo tiempo, los profesionales de la salud se quejan de sus bajos sueldos y escasas prestaciones, de la falta de servicios básicos, tales como electricidad y agua salubre, y de las malas condiciones laborales. En tales circunstancias no resulta difícil comprender por qué el país tiene dificultad a la hora de contratar y conservar al personal de salud.

\section{DEFICIENCIAS EN EL ACCESO A LAS NUEVAS VACUNAS DEL PAI}

La cobertura vacunal mundial fue promovida en 1974 mediante el PAI de la OMS. Sin embargo, en Haití comenzó al principio de los ochenta con las 6 vacunas sistemáticas de base: poliomielitis, difteria, tétanos, sarampión y tos ferina. Hoy en día, existen cuatro vacunas que se han incorporado al programa: haemophilus influenzae tipo B (Hib), hepatitis B (VHB), rubéola y fiebre amarilla. Éstas no están disponibles todavía en Haití.

El principal obstáculo para la introducción de la VHB y la Hib en el esquema vacunal sistemático del país es fundamentalmente el coste. La vacunación sistemática de un niño contra las 6 enfermedades cuesta 1 US\$. Sin embargo, las vacunas VHB y la Hib cuestan 7-13 US\$ (administración y material para la inyección no incluidos). Con la administración, llega a 20-40 US\$ por niño. Por eso, se les hace muy difícil, tanto al gobierno haitiano como a los organismos internacionales, poder incluir en el programa esas vacunas. Pero la falta de fondos es sólo parte del problema. En Haití, el sistema de inmunización carece de la capacidad necesaria para administrar las vacunas existentes y, sobre todo, para añadir las nuevas que son más costosas. Debido a la insuficiencia de los sistemas de vigilancia y notificación de enfermedades, es difícil determinar la carga de morbilidad y el coste-efectividad potencial que permitiría la incorporación de las nuevas vacunas.

\section{DEFICIENCIAS EN LA SEGURIDAD DE LA VACUNACIÓN}

En Haití, hoy en día no se puede garantizar ni la calidad ni la seguridad de las vacunas utilizadas, con lo que se pone en peligro la eficacia del programa de inmunización y la vida de los niños. Los problemas surgen sobretodo durante el transporte y almacenamiento del producto y, en el caso de algunas vacunas, durante la fase de reconstitución, en la que se mezclan con un líquido (diluyente) antes de ser administradas.

Además, los errores del programa afectan a la potencia y la inocuidad de las vacunas. La insuficiente capacitación del personal y la falta de supervisión en la manipulación, el transporte, el almacenamiento y la 
administración del producto llevan a que se suministre una vacuna que no es ni segura ni eficaz.

En algunas ocasiones se ha puesto en peligro la vida de los niños a causa de errores programáticos, que comprenden las interrupciones no detectadas en la cadena de frío (la red crítica de almacenamiento en frío formada por refrigeradores, congeladores y pequeñas neveras). Es muy frecuente, pues por la falta de electricidad la mayoría funcionan con gas propano. Muchas veces, cuando se termina el gas, no hay de reserva y el refrigerador se queda apagado con vacunas.

La reconstitución de vacunas, como la antisarampionosa, que debe mezclarse con un diluyente antes de su administración, también entraña riesgos potenciales. Ha habido casos en los que los agentes de salud han usado por error un fármaco en lugar de un diluyente, lo que ha provocado la muerte de algunos niños; en otros casos se ha administrado una vacuna sin efecto alguno por haberse añadido erróneamente demasiado diluyente. Otra situación, en la que peligra la vida de los niños a causa de una infección bacteriana, se produce cuando las vacunas reconstituidas se almacenan durante la noche y se vuelven a utilizar al día siguiente, en lugar de desecharlas después de cada día de trabajo para evitar el riesgo de contaminación.

En Haití, los errores programáticos, más que la propia vacuna, son la causa más común de incidentes adversos después de la vacunación. Uno de los principales problemas se limita en gran medida a la falta de higiene en las prácticas de inyección. Sin una respuesta rápida y efectiva a esos problemas potenciales, la confianza de la población en la inmunización desaparece de inmediato, la cobertura vacunal se desploma y se producen brotes de enfermedad, a menudo con consecuencias desastrosas. El problema es que el país no cuenta con un sistema de vigilancia eficaz que le permita detectar, investigar y responder rápidamente a los problemas asociados a la inmunización.

\section{DEFICIENCIAS DE FINANCIAMIENTO}

En Haití, ni el gobierno, ni los donantes internacionales han invertido lo suficiente en el programa de inmunización. Hay una tendencia a realizar la inmunización en forma de campañas, es decir en una época muy determinada o cada cierto tiempo, pero no de forma sistemática. Esta formar de actuar puede llevar a brotes de enfermedad capaces de poner en peligro miles de vidas. Además, existe el Fondo Rotatorio (FR) de la OPS que ofrece una línea de crédito al país, facilitándole los pedidos de vacunas. Se le ofrece la opción de poder pagar en la moneda local y el FR mejora el acceso del país al mercado internacional de vacunas, pero uno de los requisitos básicos que exige el FR es que deben de pagar las facturas dentro de un periodo de 60 días. Por supuesto, si no se cumple con este requisito, no se podrá cursar ningún pedido hasta que se hayan satisfecho todas las cantidades pendientes de pago. Es fundamental que se cumpla con el plazo de pago para reponer el capital de trabajo del FR ${ }^{9}$. Para esta tarea, la OPS "cobra" a los países un $3 \%$ del costo.

La inmunización es una de las principales responsabilidades del Estado en materia de salud pública en el país. Sin embargo, el gobierno no asigna a la inmunización recursos financieros en cantidad suficiente y de forma regular. Los recursos públicos en salud representan sólo el 0,8-1\% del PIB. La mayoría de la asignación del MSPP (57 millones US\$ en 1999, inalterado desde 1996) se gasta en sueldos. El presupuesto de inversiones depende en gran parte de la ayuda externa (49\% en 1999). Por lo tanto, las actividades se desaceleran o se detienen y la motivación del personal es baja en el país ${ }^{10}$.

La inmunización puede resultar aún más desfavorecida que otros servicios de salud en la asignación de fondos públicos. Al igual que otros servicios preventivos, la inmunización no cuenta con un grupo de beneficiarios organizados y a menudo sale perdiendo cuando compite por los escasos recursos con servicios curativos mucho más visibles. Los programas de inmunización se ven afectados por la incertidumbre en materia de financiación, la competencia tanto desde dentro como desde fuera del sector de la salud, y la creciente necesidad de apoyo financiero a medida que la cobertura se amplía y se dispone de nuevas vacunas.

En conjunto, Haití se está alejando cada vez más del logro de los beneficios de la inmunización que han conseguido los países desarrollados, y la diferencia entre ricos y pobres en materia de inmunización seguirá aumentando si ésta se deja exclusivamente a merced de los recursos del gobierno.

\section{CONCLUSIONES}

La inmunización es una de las intervenciones sanitarias esenciales que pueden ayudar a impulsar el desarrollo económico y reducir la pobreza. Los niños más pobres son los que tienen menos probabilidades de ser inmunizados y más probabilidades de morir antes de cumplir cinco años. Los que sobreviven y crecen en condiciones de pobreza extrema son los que más probabilidades tienen de verse atrapados en un círculo vicioso de desnutrición, falta de acceso a agua potable, deficiencias de saneamiento, mala salud, escolarización insuficiente y, por ende, de no lograr una plena realización personal, herencia que 
probablemente pesará también sobre la siguiente generación.

Al prevenir las enfermedades infecciosas y aumentar la esperanza de vida, la inmunización contribuye a reforzar las posibilidades de aprendizaje del niño y su capacidad de ganarse la vida como adulto, reduciendo así la pobreza y aumentando el potencial de crecimiento económico del país. Además, al combatir esas enfermedades, principal factor en las diferencias de esperanza de vida entre ricos y pobres, la inmunización puede ayudar a reducir las desigualdades en términos de salud.

La vacunación infantil es una de las intervenciones sanitarias más eficaces en relación con el costo: salva más vidas por el dinero invertido que casi ninguna otra intervención sanitaria disponible hoy en día. En promedio, el costo de inmunizar completamente a un niño con las seis vacunas tradicionales del PAl disponibles en el país se eleva a 17 US \$, incluidos los costos de entrega ${ }^{11}$, una suma muy inferior al costo del tratamiento de un niño que haya sucumbido a enfermedades prevenibles mediante la vacunación. Con las vacunas se ahorra dinero, pues por cada dólar gastado en vacunas se ahorran siete dólares en gastos médicos y 25 dólares en gastos generales relacionados con las enfermedades que se evitan con las vacunas ${ }^{12}$.

Todo el mundo reconoce el efecto saludable de las vacunas, el poder de la inmunización y la efectividad de la colaboración entre el gobierno, las Organizaciones No Gubernamentales (ONG), el sector privado y las organizaciones internacionales. Se puede esperar que Haití sabrá salvar todos los obstáculos, la violencia, la pobreza y la mala gestión y lograr con certeza una victoria sobre el enemigo común sobre el que hay que estar unidos: las enfermedades que matan inútilmente tantos niños.

\section{BIBLIOGRAFÍA}

1. The World Bank. Haiti country data profile; 2006. [acceso 9 de diciembre de 2007]. Disponible en: http://www.worldbank. org/

2. Ministère de la Santé Publique et de la Population (Haiti). Programme Elargi de Vaccination; 2007. [acceso 8 de diciembre de 2007]. Disponible en: htpp://mspp.ht/programme/lectureRubrique.php?RubriquelD $=8$

3. Organización Panamericana de la Salud. La santé: un droit pour tous; 2005. [acceso 9 de diciembre de 2007]. Disponible en: http://www.paho.org/english/d/csu/FrenchBrochure.pdf

4. Mortalité et Utilisation des Services (EMMUS IV) [Revista electrónica], Haiti 2006, [acceso 7 de diciembre de 2007]. Disponible en: http://www.measuredhs.com/pubs/pdf/FR192/ FR192\%2Epdf

5. UNICEF. La Vaccination en Haití; 2007. [acceso 10 de diciembre de 2007]. Disponible en: http://www.unicef.org/haiti/french/reallives_9903.htm

6. World Health Organization. Inmunization profile - Haiti; 2007. [acceso 6 de diciembre de 2007]. Disponible en: http://www. who.int/vaccines/globalsummary/immunization/countryprofileresult.cfm

7. World Health Organization. Immunization profile -Haiti; 2007. [acceso 9 de diciembre de 2007]. Disponible en: http://www. who.int/vaccines/globalsummary/immunization/countryprofileresult.cfm

8. UNICEF. Estado mundial de la infancia; 2007. [acceso 5 de diciembre de 2007]. Disponible en : http://www.unicef.org/spanish/sowc07/docs/sowc07_sp.pdf

9. Organización Panamericana de la Salud. Vacunas e Inmunización; 2006. [acceso 6 de diciembre de 2007]. Disponible en: http://www.paho.org/

10. Perfiles Básicos de Salud de Países en las Américas -Haití 2002. [acceso 7 de diciembre de 2007]. Disponible en: http:// www.paho.org/Spanish/DD/AIS/cp_332.htm

11. UNICEF. Le pouvoir de la vaccination; 2000. [acceso 9 de diciembre de 2007]. Disponible en: http://www.unicef.org/french/pon00/pon00_4.pdf

12. Public Health-Seattle \& King Country. Informes sobre vacunas infantil.; 2001. [acceso 11 de diciembre de 2007]. Disponible en: http://www.metrokc.gov/health/immunization/vacunasinfantil.pdf 